\title{
Improving lower extremity joint kinematics during jump landing using an automated vibrotactile biofeedback system
}

\author{
Aruni Upeksha Alahakone \\ School of Engineering, \\ Monash University Sunway Campus, \\ Jalan Lagoon Selatan, 46150 Bandar Sunway, Malaysia \\ E-mail: aruni.alahakone@gmail.com
}

\section{S.M.N. Arosha Senanayake*}

Faculty of Science,

University of Brunei,

Jalan Tungku Link, Gadong, BE1410, Brunei

E-mail: arosha.senanayake@ubd.edu.bn

*Corresponding author

\begin{abstract}
Real time motion monitoring and biofeedback is essential for efficient training, technique improvements and reducing the risk of injuries in sports training applications. This paper proposes a novel approach for measuring and improving jump landing technique based on lower extremity joint kinematics. The system consists of wireless wearable inertial sensors, a customised software for data processing and a wireless biofeedback unit including vibrotactile actuators for vibration feedback. Biofeedback is provided for knee angular measurements obtained with respect to predefined target thresholds. A drop vertical jump (DVJ) was utilised to verify the effects of real time vibrotactile feedback to improving knee flexion and abduction/adduction during the landing phase of the jump. The results confirmed the accuracy of the system for real time applications and the impact of vibrotactile biofeedback for augmenting the lower extremity joint kinematic motion during jump landing.
\end{abstract}

Keywords: inertial sensors; virtual instrumentation; data acquisition; lower extremity kinematics; jump landing; landing phase detection; vibrotactile biofeedback; motor augmentation.

Reference to this paper should be made as follows: Alahakone, A.U. and Senanayake, S.M.N.A. (2014) 'Improving lower extremity joint kinematics during jump landing using an automated vibrotactile biofeedback system', Int. J. Autonomic Computing, Vol. 2, No. 1, pp.39-53.

Biographical notes: Aruni Upeksha Alahakone became a member of IEEE in 2007. She completed her first degree in Engineering (Mechatronics) at Monash University Sunway Campus (MUSC), Malaysia in 2007, with a First Class Honours. In 2008, she received her Higher Degree by Research Scholarship from MUSC, and completed her Masters degree in Engineering (by research) under the supervision of Dr. Arosha Senanayake, in the field of human motion analysis and biomechanics. Her research experience included the development of a geographic information system for path planning and navigation of mobile objects (Final Year Project, 2007), and the development of biofeedback 
systems for sports and rehabilitation, under the project 'Bio-inspired robotics devices for sportsman screening service (BIRDSSS)' funded by Ministry of Science, Technology and Innovation (MOSTI) in 2008 to 2010. During her two years of research involvement, she managed to publish five peer reviewed publications in international journals and conferences.

S.M.N. Arosha Senanayake became a member of IEEE in 2005 and a senior member in 2006. He is currently a Senior Lecturer with Monash University Sunway Campus where he leads the research project intelligent, integrated and interactive systems (IIIS) funded by Monash University Research Funds. He is also the Project Leader of Bio-Inspired Robotics Devices for Sportsman Screening Service (BIRDSSS) under eScience Fund, Ministry of Science, Technology and Innovation (MOSTI) in 2008 to 2010. During his 20 years of research experience, he managed to publish over 105 peer reviewed publications in international conferences, journals and book chapters. He is one of the editors of three books published based on the research outcomes. He was the keynote speaker for various international conferences, and he has been invited to organise invited/special sessions for various international conferences, in particular IEEE conferences in the area of human motion reconstruction and regenerations.

\section{Introduction}

Human Motion analysis has become an essential tool for motion diagnostics in a wide range of applications involving sports, medical domain and video surveillance. The ultimate goal of motion analysis in sports is to improve technique and prevent or reduce the risk of sports injuries (McGinnis, 2005). Many researchers occupied in sports biomechanics have shown a great interest in kinematics involved in jump landing tasks in sports activities (Louw and Grimmer, 2006; Urabe et al., 2005; Nagano et al., 2009). This is due to the high risk of anterior cruciate ligament (ACL) injury that can occur due to poor landing postures followed by athletes (Noyes et al., 1983). Identifying and understanding biomechanical risk factors associated with jump landing can aid in developing preventive measures to be followed during sports training (Louw and Grimmer, 2006). There is general consensus in the literature that ACL injuries can be minimised by training athletes to increase knee flexion and reduce knee abduction/adduction (abd/add) during landing (Munro et al., 2008; Hewett et al., 2005).

However, identifying an approach to ensure that athletes participating in a landing training programme achieve desired knee kinematics is challenging. This is where the role of biofeedback systems is realised. A biofeedback system typically incorporates a sensory device, a restitution device that can convert the biofeedback information to the subjects and a processing system that can perform computations, decision making and control of the input/output devices in the system (Dozza, 2006). Restitution mechanisms involve devices that produce auditory, visual or tactile feedback to the users in response to their measured movements. Many studies have investigated the use of verbal or auditory and visual feedback to indicate knee kinematics and ground reaction forces involved during landing. Cowling et al. (2003) investigated the effect of verbal instruction to improve muscle activity and reduce the risk of ACL injury during landing. 
Saggital plane kinematics, ground reaction forces and electromyography data were collected during landing, and verbal instructions on knee flexion and muscle activity was provided at the end of jumps. The results demonstrated a significant increase in knee flexion angle during landing at initial contact and peak contact. However, feedback received after landing tasks may limit the extent of improvement in landing kinematics, and may impose a larger effort on the subjects to concentrate and remember the feedback instruction during their performance. Munro et al. (2008) presented a more practical approach for improving knee flexion angle at landing using audio biofeedback, with respect to a predefined training target. However, the measurement device was not suitable to discriminate minor changes of knee flexion $\left(<10^{\circ}\right)$ and had limited use when damped by sweat and humid environmental conditions. Noyes et al. (2005) and Onate et al. (2001, 2005) described the impact of visual feedback using video tape information for improving knee separation during landing and increasing knee flexion and reducing peak vertical forces during landing respectively.

However, auditory and visual feedback can interfere with a subject's visual or acoustic dependency affecting their performance. Furthermore, feedback occurring before or after a training event may not assist in improving the motion during the performance.

Tactile feedback is an ideal counter-measure opposed to auditory and visual feedback systems. Tactile feedback systems exist in electrotactile, thermal and vibrotactile modalities (Alonso et al., 2008). The concept of tactile technology is based on the skin's ability to communicate information through these modalities. Vibrotactile systems are identified to be safer on human skin and have the ability to decipher feedback information in a simple, realistic manner (Alahakone and Senanayake, 2009). The effectiveness of vibrotactile systems in real time applications have been realised by many researchers. A few typical paradigms are presented in Van Erp et al. (2006), Lieberman and Breazeal (2007), Jacobs (2008), Kalisvaart et al. (2004) and Alahakone and Senanayake (2010). Their suitability in such applications provide evidence that landing training programmes can benefit from real time vibrotactile feedback for lower extremity motion augmentation during dynamic landing tasks.

The main emphasis of this research is the design, implementation and testing of a real time biofeedback system for monitoring and improving jump landing movements during sports training. The system consists of two inertial sensors for the measurement of knee joint kinematics, a vibration stimulus module to provide biofeedback compared to reference thresholds and a custom developed user interface that performs data acquisition, processing, biofeedback generation and data analysis. To complement the training sessions, video recording of movements performed is incorporated in the system via a web camera. Data acquisition from inertial sensors and biofeedback signalling is performed wirelessly enabling the system devices to be completely wearable and convenient to the users. A drop vertical jump (DVJ) protocol was utilised to verify the accuracy of the system and the effect of immediate biofeedback to improve landing technique. Laboratory results demonstrate the viability of the system in terms of data reliability, real time performance in which feedback is provided during the motion opposed to before or after a training session and the significance of vibrotactile feedback, resolving the inconvenience and interference to performance that is frequently encountered in auditory/visual biofeedback systems. 


\section{Hardware/software co-design architecture}

The integrated hardware/software co-design employs four main modules, a sensing module, a host PC, vision module and remote biofeedback module. The sensing module includes two inertial sensors for knee angle measurements. The host PC running the LabVIEW-based system software performs data validations, landing phase detection and biofeedback generation with respect to target thresholds. Concurrently, knee kinematics is graphically represented and biofeedback activation is depicted via a visual display on the interface. The feedback commands generated are transmitted wirelessly to the remote module which performs the activation of the corresponding actuators based on the received commands. In this manner, a closed loop control is achieved in which immediate feedback is conveyed in correspondence to current knee kinematics during landing tasks.

The vision module includes a USB web camera connected to the system software that continuously captures visual information for video recording. Both kinematic and video data captured throughout a training session can be saved for later referencing. The user interface employs data analysis functionalities for revisiting the recorded datasets for thorough observations and long term analysis. Figure 1 depicts the system architecture of the implementation.

Figure 1 System architecture

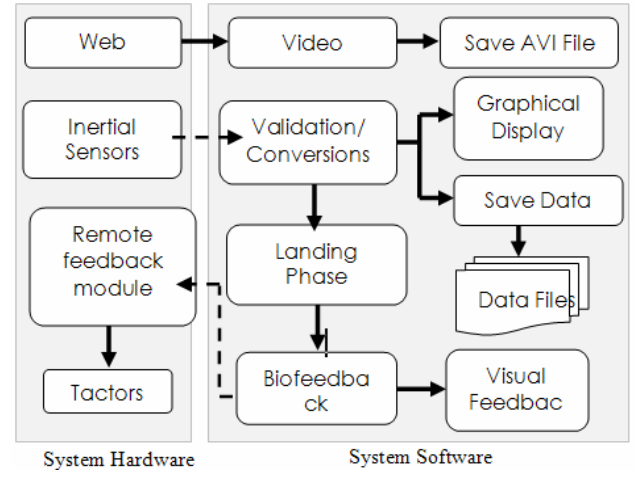

\section{System hardware}

The system hardware incorporates inertial sensors, a USB web camera and a vibrotactile control circuit including tactile actuators, each of which is described below.

\subsection{Inertial sensors}

The tri-axial inertial sensors used in this study are the microstrain's inertia link. The sensor unit is compact in size $(41 \mathrm{~mm} \times 63 \mathrm{~mm} \times 24 \mathrm{~mm})$ and weight $(39 \mathrm{~g})$, and each unit involves a base station that connects to the host computer for wireless data acquisition. The highlight of this unit is its ability to stream reliable packetised data with high data rates of up to $100 \mathrm{~Hz}$. The unit supports orientation measurements in roll, pitch 
and yaw directions and this study utilises measurements of roll and pitch axes to obtain hip and shank angular displacements from which knee kinematics are computed for immediate biofeedback at landing.

\subsection{Creative web camera}

A simple web camera from Creative (VF0080) with a USB interface was used for video capture of the exercises performed. The capture speed of the web camera is about 30 frames per second.

\subsection{Feedback control circuit development}

A tactile control circuit was built in the system to wirelessly receive commands from the host computer to activate the corresponding tactile actuators. The circuit involves a Cypress CY8C27443 8 bit microcontroller, a radio frequency transceiver (ER 400TRS), audio amplifiers (MC34119) that behaves as motor drivers for the actuators, VBW32 tactile actuators, $9 \mathrm{~V}$ batteries for power supply and $\mathrm{L} 7805 \mathrm{CV}$ voltage regulators. An RF transceiver unit interfaced via a serial line to the host computer wirelessly transmits the commands to the remote unit. The on-board processor in the remote unit is preprogrammed to continuously listen to incoming tactile commands, and when a command is received, output a $250 \mathrm{~Hz}$ square wave signal to the corresponding tactor driver using pulse width modulation (PWM). The drivers amplify the input signal and activate the corresponding tactile actuators at the given instance.

The tactile actuators employed in this development are Tactaid VBW32 from Audiological Engineering Corporation, USA. Tactile actuators are small, light weighted elements that produce a vibration when powered. VBW32 has a compact size of $1^{\prime} \times 0.73^{\prime} \times 0.42^{\prime}$, weighs $6.5 \mathrm{~g}$ and has a resonant frequency of $250 \mathrm{~Hz}$ which is ideal to be recognised on human skin. The actuators have very high ring-up and ring-down times which makes them ideal for real time applications.

\section{System software}

The software framework built into the system was developed using LabVIEW Virtual Instrumentation which performs data capture and validation, landing phase detection and biofeedback signalling during training sessions which are made accessible via an interactive graphical user interface (GUI). The following illustrates the sequential procedure incorporated for providing biofeedback during dynamic landing tasks.

- computation of knee flexion and knee abd/add angles from hip and shank angular displacements for each incoming reading

- landing phase detection based on discernible knee kinematic properties occurring at ground contact

- activation of biofeedback when landing phase is detected based on predefined target thresholds for knee flexion and abd/add

- detection of end of jump and deactivating biofeedback signalling. 
The subsequent sections describe in detail the features and functionalities incorporated in the GUI and the algorithms implemented for landing phase detection and biofeedback generation respectively.

\subsection{Graphical user interface}

\subsubsection{Hardware settings and calibration tools}

The system hardware configurations and device calibrations can be performed via the interface without requiring using any external software. Via calibration and hardware settings tools, sensor placements based on corresponding axes and hardware reconfigurations such as wireless channel selection, baudrate settings, power, etc., can be performed.

\subsubsection{Subject details}

The subject details entry includes subject name, age, gender, protocol/activity to be monitored, time, date and the test operator's name. This information will be saved along with kinematic data obtained for each activity after every training session.

\subsubsection{Real time monitoring and biofeedback tools}

Real time monitoring and biofeedback functions include the selection of suitable target thresholds to be utilised for biofeedback activation, selection of axis of movement, number of actuators to be used, graphical representation of knee kinematics, visual display of biofeedback activation, video display of the training sessions and saving data for later referencing. The axis selection enables monitoring movements about a single plane or multiple planes of motion. The numbers of actuators vary in correspondence to the axis selection. For the experiment discussed in this paper, multi axis motion is utilised to monitor both knee flexion and knee abd/add angles at landing, and biofeedback is provided using two actuators corresponding to each axis of movement.

The selection of threshold limits is specific to each individual and the training protocol performed. Via the interface, users can enter the suitable ranges with regard to the training to be conducted. During a DVJ, a simple algorithm identifies the landing phase and initiates the biofeedback activation algorithm which wirelessly triggers the tactile actuators in correspondence to the target ranges for knee flexion and knee abd/add. Concurrently, the current motion with respect to the thresholds is depicted via a visual display on the interface. While the subjects receive vibrotactile feedback, the visual display provides feedback to the system operators during monitoring a training session.

Kinematic measurements gathered during an activity session are saved as text documents for later referencing. Video capture is also facilitated via this frame, which initiates video capturing as soon as a training session is started. Recorded video data can also be saved for later referencing.

\subsubsection{Data analysis tools}

Data Analysis tools are dedicated for post analysis of the data obtained during a training session (Figure 2). The interface supports graphical and video representations of the data recorded. Furthermore, when a recorded dataset is imported, the corresponding subject 
details are presented as well which identifies the test protocol, the axis and the threshold limits used.

Figure 2 Data analysis tools

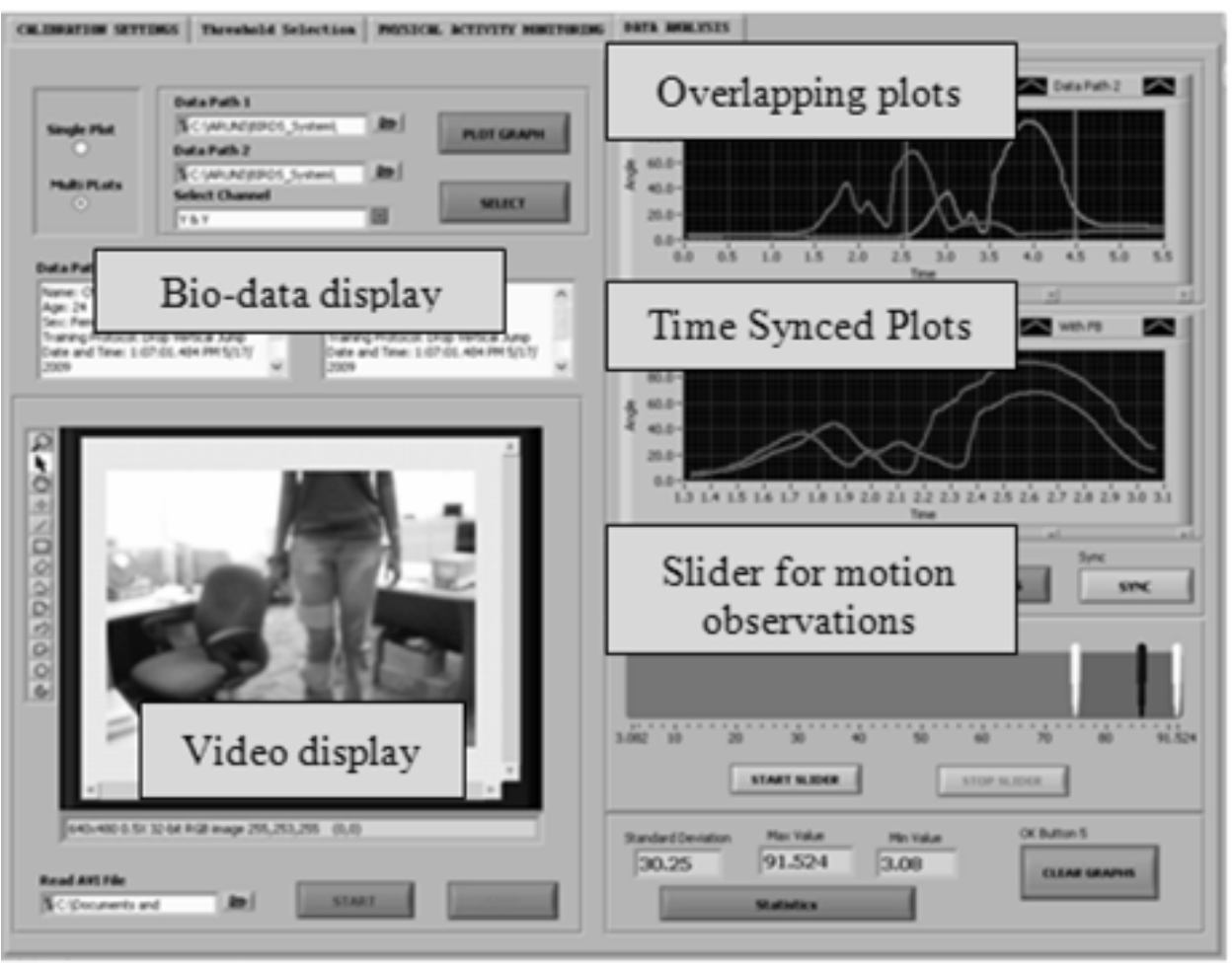

Comparisons between different planes of motion, or different session records can be performed using overlapping graphical representations and time synchronisation tools. These functionalities are beneficial to perform a detailed analysis of a particular individual's performance and monitor his/her improvements over a period of time.

To compliment the kinematic data, video recordings saved can be imported to be visualised along with training session records.

\subsection{Landing phase detection and biofeedback generation}

To provide biofeedback during landing, a landing phase detection algorithm was developed. The initiation of the landing phase was distinguished by analysing the hip and shank flexion trajectories of a DVJ. These attributes are observed to be uniquely discernible over the course of a DVJ. The instant at which ground contact occurs was further clarified using a shoe insole consisting of four force-sensitive resistors (FSR) (Senanayake and Senanayake, 2009).

Figure 3 depicts the hip and shank angular displacement trajectories obtained for a representative DVJ and the instances at which initial ground contact at landing and the end of the jump is reached. The landing phase detection algorithm takes into account the angular values of the shank, hip and the negative-to-positive gradient transition of the 
shank angle between the current and the previous measurement occurring immediately at ground contact. During a DVJ training session, the algorithm continuously checks if the hip angular displacement falls within a defined threshold limit $\left(\theta_{h 1} \leq \theta_{h} \leq \theta_{h 2}\right)$, shank angular displacement falls within a defined threshold limit $\left(\theta_{s 1} \leq \theta_{s} \leq \theta_{s 2}\right)$ and the shank angle value undergoes a negative-to-position gradient transition. If all conditions are reached, the landing phase is detected, and the biofeedback activation algorithm is started. Figure 4 illustrates the sequence of the signals being processed for landing phase detection and biofeedback activation. Similarly, the end of a landing phase is detected when a subject reaches the upright position after the jump where the hip and shank angular values fall to near-zero values, $\left(\theta_{H 1} \leq \theta_{\text {hip }} \leq \theta_{H 2}\right)$ and $\left(\theta_{S 1} \leq \theta_{\text {shank }} \leq \theta_{S 2}\right)$, as that obtained at the initial position. Once a landing phase is detected, the algorithm continuously checks for the initial conditions in the shank and hip angles, and when reached, the end of a landing phase is detected and the biofeedback activation algorithm is terminated.

Figure 3 Hip and Shank angular displacement trajectories during a DVJ indicating ground contact and end of jump (see online version for colours)

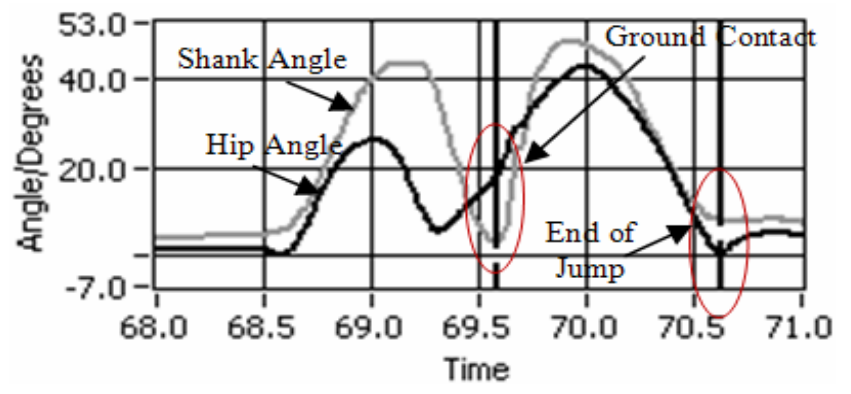

Figure 4 Signal processing sequence for landing phase detection and biofeedback activation

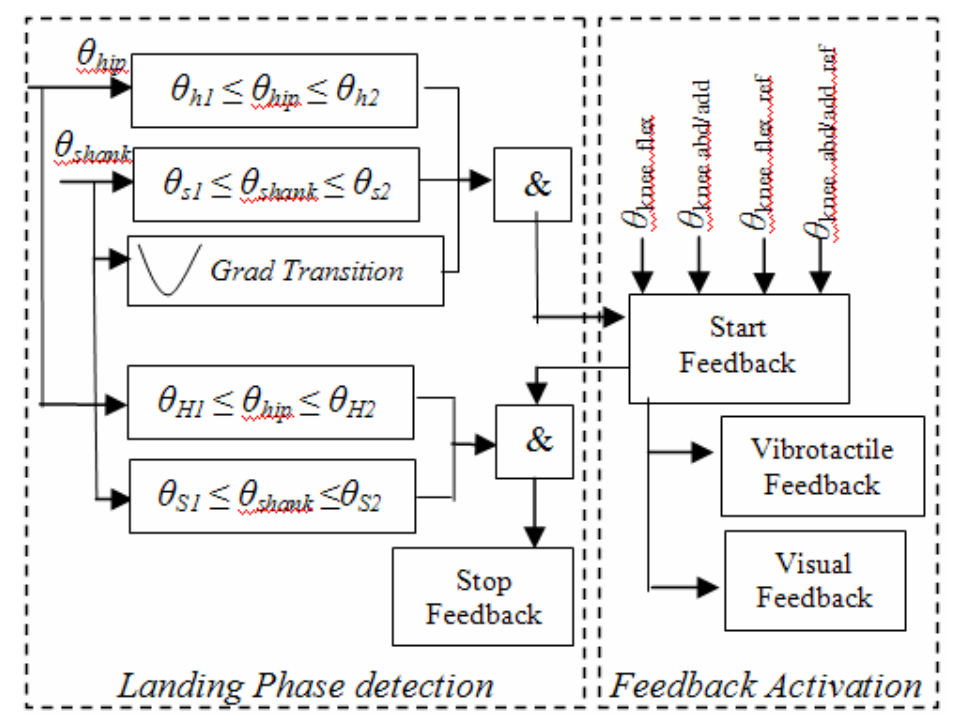


During a landing phase, the system evaluates the incoming knee flexion and knee abd/add measurements against the defined thresholds for biofeedback activation. If target knee kinematics is reached, both actuators are triggered indicating that required knee flexion and knee abd/add was attained at landing. Similarly, if only a single condition is achieved, the corresponding actuator is triggered, and if both conditions are not satisfied, actuators remain inactive.

Biofeedback signals are immediately transmitted wirelessly to the remote module that performs the activation of tactile actuators. A simple ASCII protocol is developed to identify the required actuators to be activated based on the output produced by the biofeedback algorithm. The processing unit in the remote module is programmed to acquire data transmitted from the host and activate the corresponding actuators with a strong pulse rate to convey landing knee kinematics to the subjects.

\section{Experimental setup}

\subsection{Test protocol}

The test protocol involved a DVJ from a height of about $30 \mathrm{~cm}$, to monitor and provide biofeedback for knee flexion and knee abd/add angles measured during landing. A threshold range of 75 to 95 degrees for peak knee flexion and abd/add range of \pm 3.5 degrees during landing were utilised during the trials for biofeedback (Hewett et al., 2005). When the knee flexion and abd/add angles reached the given thresholds, vibrotactile biofeedback was activated to indicate to the subjects that proper knee angle kinematics have been reached.

Figure 5 Sensor and tactor placements

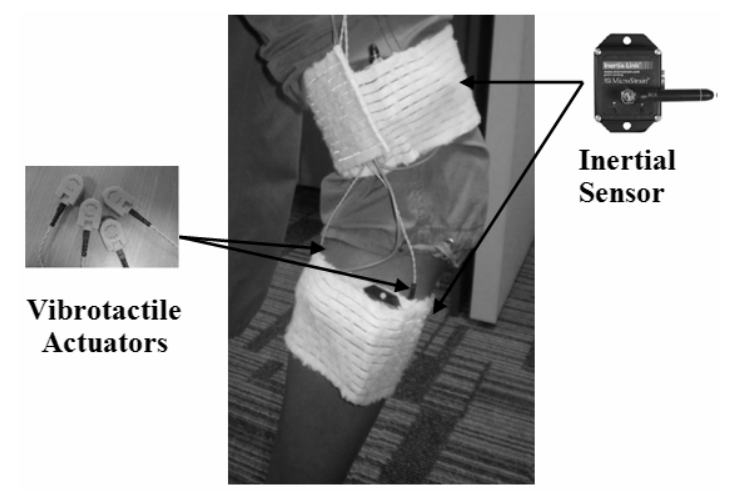

\subsection{Sensor and tactor placement}

The two inertial sensors were placed each on the thigh and shank of the test subjects. Both sensors were oriented to produce knee flexion about the $\mathrm{Y}$ axis and knee abd/add about the $\mathrm{X}$ axis. Flexion angle was computed using the flexion measurements obtained from the thigh and shank, and abd/add angle was obtained from the sensor placed on the shank (Figure 5). Two tactile actuators were utilised; each placed at the back of the shank 
for flexion biofeedback and at the side of the shank for abd/add biofeedback. Sensors and actuators were securely placed using elastic body straps.

\subsection{Experimental procedure}

A total of 5 female subjects participated in the experiment as females are identified to be more susceptible to injuries during landing compared to their male counterparts (Hewett et al., 2005). All subjects were young, healthy individuals with an average age of 23.2 years and standard deviation (SD) of 0.84 years. Prior to the initiation of the test trials, the subjects were instructed on how to perform the DVJ. Biofeedback was also introduced during the familiarisation period to become accustomed to the nature of feedback. After the familiarisation period, each subject performed the DVJ without the feedback system for five trials, and their knee angle kinematics was monitored by the system. Once completed, the biofeedback devices were mounted on the subjects, and the same regimen was carried out with each subject completing five trials. It was observed that the subjects continued to orient their knees at landing until the feedback was delivered. All trials were visualised in real time in the user interface and all data gathered were saved for further analysis.

\subsection{Statistical analysis of experimental results}

To evaluate the impact of vibrotactile biofeedback delivered during jump landing, the maximum knee flexion angle reached and the root mean square (RMS) of the knee abd/add angle during the landing phase was computed. The two parameters were averaged over all trials for each subject and paired t-tests were utilised to evaluate the statistical significance of vibrotactile feedback for improving landing knee kinematics. As a low sample size was used, Shapiro-Wilk normality tests were performed to confirm normality for knee flexion and abd/add data prior to using paired t-tests. The threshold for statistical significance was set as 0.05 .

\section{Results}

\subsection{System reliability for biofeedback activation}

Figures 6(a) to 6(e) depicts the output of the landing phase detection algorithm, knee flexion angle, knee flexion biofeedback signal activation, knee abd/add angle and knee abd/add biofeedback signal activation for a representative DVJ trial. It is clearly observed from Figure 6(a) that the instances at which initial ground contact and the termination of the jump occurred were accurately detected by the landing phase detection algorithm. With regard to the defined control parameters for knee flexion and knee abd/add, Figure 6(c) and 6(e) clearly illustrate the instances at which biofeedback signals were triggered during the landing phase to indicate knee orientation to the subject. As shown in Figure 6(c), for knee flexion biofeedback, the activation of feedback occurred as soon as the knee flexion angle reached the training target range during the landing phase indicating that required flexion was achieved. Similarly, as depicted in Figure 6(e) for knee abd/add, feedback was triggered immediately at landing as the knee abd/add remained within the training target range. At peak landing however, a knee adduction 
beyond the target range is displayed during which biofeedback was switched off indicating that required knee abd/add has not been reached. It is clearly observed that no misses in the biofeedback activation was reported during the test and feedback was immediately triggered without the occurrence of any significant latency. The latency involved would be the soft real time processing delays which are negligible to be perceived by subjects.

Figure 6 (a) Landing phase detection, (b) knee flexion plot during DVJ, (c) biofeedback activation for knee flexion, (d) knee abd/add plot during DVJ and (e) biofeedback activation for knee abd/add

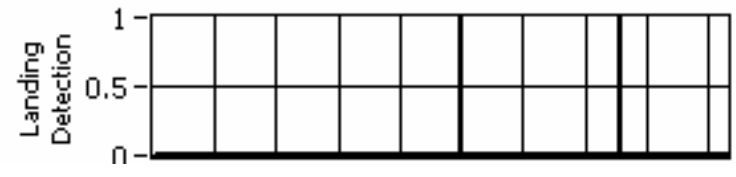

(a)

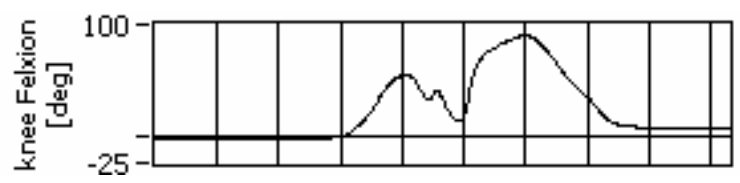

(b)

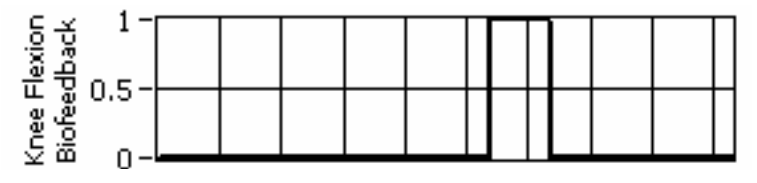

(c)

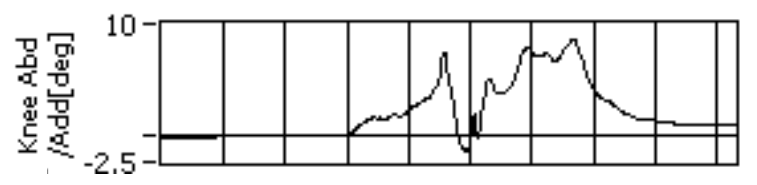

(d)

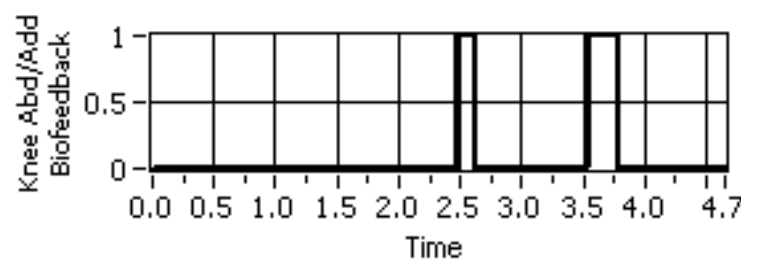

(e)

\subsection{Effects of biofeedback for motion augmentation}

Table 1 illustrates the peak knee flexion and mean knee abd/add measurements obtained for each subject for the two conditions with and without biofeedback, and the corresponding improvement ratio achieved during feedback training. 
As depicted in Table 1, all subjects reported an increase in knee flexion angle during landing when biofeedback was in use, with Subjects 3 to 5 reporting an improvement above 50\%. This implies that during training with biofeedback, subjects were able to interpret clearly the information provided via vibration stimuli and were capable of applying the feedback provided to improve knee flexion angle during landing.

Table 1 Maximum knee flexion and RMS knee abd/add during landing for all subjects in the DVJ

\begin{tabular}{lccccccc}
\hline \multirow{2}{*}{$\begin{array}{l}\text { Subject } \\
\text { no. }\end{array}$} & \multicolumn{3}{c}{ Knee flexion } & & \multicolumn{3}{c}{ Knee abd/add } \\
\cline { 2 - 3 } \cline { 7 - 8 } & Without & With & Improvement & & Without & With & Improvement \\
ratio $(\%)$ & & & $F B\left({ }^{\circ}\right)$ & $F B\left({ }^{\circ}\right)$ & ratio (\%) \\
\hline Subject 1 & 72 & 84.3 & 17.08 & & 3.75 & 4.43 & -18.13 \\
Subject 2 & 70.8 & 94 & 32.77 & & 6.54 & 4.53 & 30.73 \\
Subject 3 & 52.67 & 82.9 & 57.40 & & 4.83 & 5.67 & -17.40 \\
Subject 4 & 50.87 & 92.4 & 81.64 & & 2.54 & 2.22 & 12.60 \\
Subject 5 & 54.88 & 91.61 & 66.93 & & 3.79 & 2.51 & 33.77 \\
\hline
\end{tabular}

For Knee abd/add during landing, Subjects 2, 4 and 5 have reported an improvement with reduced knee abd/add with the inclusion of biofeedback with the highest improvement reported by Subject 5 as $33.8 \%$. Subjects 1 and 3 did not improve knee abd/add angle during landing with the use of biofeedback. This may have been due to the facts that these subjects found it difficult to concentrate on simultaneous delivery of feedback signals for both knee flexion and abd/add or the inability to interpret the information provided through feedback in an effective manner to improve performance. Figure 7(a) and 7(b) depict the overall means of the RMS angles obtained for knee kinematics over the ten trials for each condition.

The overall mean knee flexion angle and SD for all subjects over all trials was $60.24 \pm 10.3$ and $89.04 \pm 5.07$ respectively. This increase was statistically significant at $\mathrm{p}<0.05$ (0.00251) producing an overall improvement of $47.8 \%$. This implies that while training with biofeedback, subjects continued to flex their knees at landing until the feedback signal was delivered indicating that the required knee flexion was reached. The mean RMS knee abd/add angle and SD for all subjects over all trials was $4.29 \pm 1.50$ and $3.87 \pm 1.46$ respectively. This reduction in knee abd/add angle was not statistically significant at $\mathrm{p}<0.05$, however the overall reduction of knee abd/add angle by $9.8 \%$ implies that biofeedback has contributed to reducing knee abd/add angle during landing opposed to no feedback.

The results clearly demonstrate the impact of real time vibrotactile biofeedback for improving knee kinematics during landing. Improved knee flexion and knee abd/add angles obtained during landing suggest that subjects were able to interpret the information provided via vibration stimuli to improve knee kinematics at landing. Increase in knee flexion angle was significant with the inclusion of biofeedback. Although not statistically significant, knee abd/add angle was reduced with the use of biofeedback, which was consistent with the idea that feedback has contributed to improving knee abd/add angle during landing. However, if a broader training target was used or if tested on a larger sample size, the impact of biofeedback for knee abd/add angle would have been more prominent. 
None of the subjects had any difficulty in learning the nature of feedback and was easily accustomed to the wearable devices and the information provided by them. No subject reported any discomfort during the tests and all participants were able to continue the experiments throughout the due course.

Figure 7 (a) Mean knee flexion angle computed with and without biofeedback over ten trials (b) mean knee abd/add angle computed with and without biofeedback over ten trials

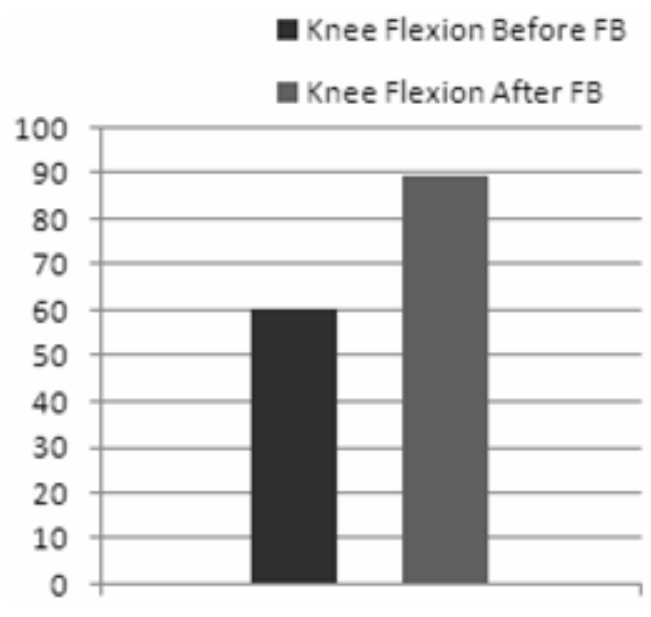

(a)

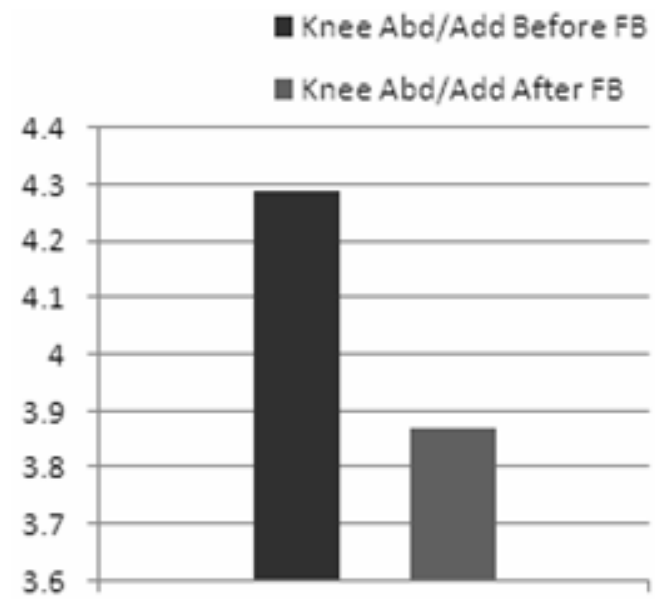

(b)

The developed biofeedback prototype for monitoring and improving knee kinematics during landing is a novel approach in terms of its hardware/software co-design architecture, real time functionality and the use of vibrotactile feedback opposed to conventional audio and visual feedback modalities. The software tools embedded allows the system operation to be customised to suit the requirements of different individuals and tasks, eliminating the applicability of the system to a single specific application. The IGUI provides informative human computer interaction for the system 
users incorporating real time data visualisation, video display, visual feedback, and supports data recording capabilities for post training data evaluations.

\section{$7 \quad$ Future work and conclusions}

This paper discussed the implementation of a novel approach for monitoring and improving knee kinematics associated with DVJ landing. System devices employed met the requirements of a real time biofeedback system in terms of wearability, portability, reliable data transfer and accurate feedback signal activation. The integrated hardware/software co-design employed in the system provides comprehensive functions and tools required for biofeedback systems within one interface, including device hardware reconfigurations, data acquisition, representations, real time biofeedback generation and data analysis features. Biofeedback control parameters are reconfigurable via the interface providing the flexibility for the system to be customised for each individual. Initial laboratory experiments conducted with young, healthy subjects confirmed the validity and reliability of the system in terms of real time performance and the impact of vibrotactile biofeedback training. Further research will be concentrated on expanding the system functionality to be applicable for different types of jump activities accessing whether augmented landing techniques and postures practiced during biofeedback training could be retained and could reduce the risk of injury during jump landing tasks.

\section{References}

Alahakone, A.U. and Senanayake, S.M.N.A. (2009) 'Vibrotactile feedback systems: current trends in rehabilitation, sports and information display', Proceedings of the 2009 IEEE/ASME International Conference on Advanced Intelligent Mechatronics (AIM), pp.1148-1153.

Alahakone, A.U. and Senanayake, S.M.N.A. (2010) 'A real time system with assistive feedback for postural control in rehabilitation', IEEE Transactions on Mechatronics (TMECH), Vol. 15, No. 2, pp.226-233.

Alonso, M.A.G., Vexo, F. and Thalmann, D. (2008) Stepping Into Virtual Reality, A Practical Approach, Springer, London.

Cowling, E.J., Steele, J.R. and McNair, P.J. (2003) 'Effect of verbal instructions on muscle activity and risk of injury to the anterior cruciate ligament during landing', Br. J. Sports Med, Vol. 37, No. 2, pp.126-130.

Dozza, M. (2006) 'Biofeedback systems for human postural control', $\mathrm{PhD}$ thesis in BioEngineering, Department of Electronics, Computer Science and Systems, University Di Bologna.

Hewett, T.E. et al. (2005) 'Biomechanical measures of neuromuscular control and valgus loading of the knee predict anterior cruciate ligament injury risk in female athletes', The American Journal of Sports Medicine, Vol. 33, No. 4, pp.492-501.

Jacobs, M. (2008) 'Design and recognition of tactile feedback patterns for snowboarding', Diploma thesis at the Media Computing Group, Computer Science Department, RWTH Aachen University.

Kalisvaart, S.H., Lechner, E.M.C.G. and Lefeber, F.J. (2004) 'System for monitoring and coaching of sportsmen', European Symposium on Ambient Intelligence (EUSAI), Vol. 3295, pp.88-91. 
Lieberman, J. and Breazeal, C. (2007) 'TIKL: development of a wearable vibrotactile feedback suit for improved human motor learning', IEEE Transactions on Robotics, Vol. 23, No. 5, pp.919-926.

Louw, Q. and Grimmer, K. (2006) 'Biomechanical factors associated with the risk of knee injury when landing from a jump', Soft African Journal of Sports Medicine, Vol. 18, No. 1, pp.18-23.

McGinnis, P.M. (2005) Biomechanics of Sport and Exercise, published by Human Kinetics, USA.

Munro, B.J., Campbell, T.E., Wallace, G.G. and Steele, J.R. (2008) 'The intelligent knee sleeve: a wearable biofeedback device', Sensors and Actuators B: Chemical, Vol. 131, No. 2, pp.541-547.

Nagano, Y., Ida, H., Akai, M. and Fukubayashi, T. (2009) 'Biomechanical characteristics of the knee joint in female athletes during tasks associated with anterior cruciate ligament injury', The Knee, Vol. 16, No. 2, pp.153-158.

Noyes, F.R., Barber-Westin, S.D., Fleckenstein, C., Walsh, C. and West, J. (2005) 'The drop-jump screening test, difference in lower limb control by gender and effect of neuromuscular training in female athletes', American Journal of Sports Medicine, Vol. 33, No. 2, pp.197-207.

Noyes, F.R., Mooar, P.A. and Matthews, D.S. (1983) 'The symptomatic anterior cruciate-deficient knee. I. The long-term functional disability in athletically active individuals', J. Bone Joint Surg., Vol. 65A, No. 2, pp.154-162.

Onate, J.A., Guskiewicz, K.M. and Sullivan, R.J. (2001) 'Augmented Feedback reduces jump landing forces', J. Orthop. Sports Phys. Ther., Vol. 31, No. 9, pp.511-517.

Onate, J.A., Guskiewicz, K.M., Marshall, S.W., Giuliani, C., Yu, B. and Garrett, W.E. (2005) 'Instruction of jump-landing technique using videotape feedback: altering lower extremity motion patterns', American Journal of Sports Medicine, Vol. 33, No. 6, pp.831-842.

Senanayake, C.M. and Senanayake, S.M.N.A. (2009) 'Human assisted tools for gait analysis and intelligent gait phase detection', Conference on Innovative Technologies in Intelligent Systems \& Industrial Applications (CITISIA), pp.230-235.

Urabe, Y., Kobayashi, R., Sumida, S., Tanaka, K., Yoshida, N., Nishiwaki, G.A., Tsutsumi, E. and Ochi, M. (2005) 'Electromyographic analysis of the knee during jump landing in male and female athletes', The Knee, Vol. 12, No. 2, pp.129-134.

Van Erp, J.B.F., Saturday, I. and Jansen, C. (2006) 'Application of tactile displays in sports: where to, now and when to move', Proceedings of EuroHaptics, Vol. f1, pp.1-5. 